Ram Niwas', Aneesa Shahul S', M K Garg', Vijaya Lakshmi Nag ${ }^{3}$, Pradeep Kumar Bhatia ${ }^{4}$, Naveen Dutt', Nishant Chauhan', Jaykaran Charan ${ }^{5}$, Shahir Asfahan', Praveen Sharma ${ }^{6}$, Pankaj Bhardwaj ${ }^{7}$ Mithu Banerjee ${ }^{6}$, Pawan Garg $^{8}$, Binit Sureka ${ }^{8}$, Gopal Krishna Bohra ${ }^{2}$, Maya Gopalakrishnan', Sanjeev Misra9

'Department of Pulmonary Medicine, All India Institute of Medical Sciences Jodhpur, India

${ }^{2}$ Department of General Medicine, All India Institute of Medical Sciences Jodhpur, India

${ }^{3}$ Department of Microbiology, All India Institute of Medical Sciences Jodhpur, India

${ }^{4}$ Department of Anaesthesiology \& Critical Care, All India Institute of Medical Sciences Jodhpur, India

${ }^{5}$ Department of Pharmacology, All India Institute of Medical Sciences Jodhpur, India

${ }^{6}$ Department of Biochemistry, All India Institute of Medical Sciences Jodhpur, India

'Department of Community Medicine and Family Medicine, All India Institute of Medical Sciences Jodhpur, India

${ }^{8}$ Department of Diagnostic \& Interventional Radiology, All India Institute of Medical Sciences Jodhpur, India

${ }^{9}$ Department of Surgical Oncology, All India Institute of Medical Sciences Jodhpur, India

\title{
Clinical outcome, viral response and safety profile of chloroquine in COVID-19 patients - initial experience
}

\begin{abstract}
Introduction: Chloroquine and its analogues are currently being investigated for the treatment and post exposure prophylaxis of COVID-19 due to its antiviral activity and immunomodulatory activity.

Material and methods: Confirmed symptomatic cases of COVID-19 were included in the study. Patients were supposed to receive chloroquine (CO) $500 \mathrm{mg}$ twice daily for 7 days. Due to a change in institutional protocol, initial patients received chloroquine and subsequent patients who did not receive chloroquine served as negative controls. Clinical effectiveness was determined in terms of timing of symptom resolution and conversion rate of reverse transcriptase polymerase chain reaction (RT-PCR) on day 14 and day 15 of admission.

Results: Twelve COVID-19 patients formed the treatment arm and 17 patients were included in the control arm. The duration of symptoms among the $\mathrm{C} Q$ treated group $(6.3 \pm 2.7$ days) was significantly ( $p$-value $=0.009$ ) lower than that of the control group ( $8.9 \pm 2.2$ days). There was no significant difference in the rate of RT-PCR negativity in both groups. 2 patients out of 12 developed diarrhea in the CQ therapy arm.

Conclusion: The duration of symptoms among the treated group (with chloroquine) was significantly lower than that of the control group. RT-PCR conversion was not significantly different between the 2 groups.
\end{abstract}

Key words: COVID-19, chloroquine, viral conversion, safety profile

Adv Respir Med. 2020; 88: 515-519

\section{Introduction}

COVID-19 was first detected in the Wuhan province of China and it has since traversed all natural barriers and spread to all continents of the world. COVID-19 is caused by the SARS-CoV-2 virus belonging to the family of Corona viruses and was previously referred to as the $2019 \mathrm{n}-\mathrm{CoV}$. Within the limits of our current understanding, person to person spread of COVID-19 occurs through droplets and fomites [1-3]. The incubation period is presumed to be 2 to 14 days. The spectrum of disease ranges from a mild self-limiting upper respiratory infection to Multi-Organ Dysfunction Syndrome [3-5].

Chloroquine (CQ) and hydroxychloroquine have been used in the management of COVID-19 due to their theoretical antiviral activity [6-9]. China's National Health Commission had reported that CQ was associated with reduced

Address for correspondence: Ram Shahul Niwas, Department of Pulmonary Medicine, All India Institute of Medical Sciences Jodhpur, India; e-mail: rniwasaïms@gmail.com DOI: $10.5603 /$ ARM.a2020.0139

Received: 26.06.2020

Copyright (C) 2020 PTChP

ISSN 2451-4934 
progression of the disease and a decreased duration of symptoms [10,11].

In light of the above, we considered it necessary to undertake a study to describe the clinical course and treatment outcomes in COVID-19 patients treated with CQ. This was especially necessary in a malaria-endemic country like India where CQ is easily available and doctors have extensive experience using it.

\section{Objectives}

Our primary objective was to study the clinical and viral effectiveness of CQ in COVID-19 positive patients. The secondary objective was to study its safety profile.

\section{Material and methods}

\section{Study design}

This was an observational case-control study done at a COVID referral center in western India.

\section{Methodology and participants}

All COVID-19 positive patients were hospitalized as per protocol. Symptomatic hospitalized RT-PCR confirmed COVID-19 patients ( $>18$ years old) were included in the study after providing informed written consent. The study protocol was approved by the Institute Ethics Committee vide. AIIMS/IEC/2020-21/1035. Originally designed to be a descriptive observational study, we initially recruited patients who received CQ as per our protocol. Due to changes in institutional guidelines, more patients could not be recruited into the CQ arm. Hence, patients who did not receive CQ served as controls in this study. Patients were excluded if they had a known hypersensitivity to CQ, cardiac arrhythmias, history of retinopathy, glucose-6-phosphate dehydrogenase deficiency, a prolonged corrected QT interval more than 450 milliseconds, and baseline aspartate transaminase, alanine transaminase levels $>5$ times the upper limit of normal in the blood. Pregnant patients, lactating mothers, bone marrow transplant recipients, patients with stage 4 chronic kidney disease, and patients on maintenance hemodialysis were also excluded from the study.

The demographic details, clinical findings, and investigation results were recorded in a pre-designed proforma. A detailed history including symptoms and their duration was recorded. Study participants in the CQ group received CQ $500 \mathrm{mg}$ twice daily for 7 days.
Repeat RT-PCR was done on day 14 and 15. Clinical outcomes included the number of days of symptoms, RT-PCR conversion, and disease-related complications. The safety profile of therapy was determined in terms of the incidence of therapy-related complications.

\section{Statistical methods}

Data was analyzed using Python 3.6. Quantitative data were presented as means and standard deviations (SD). Qualitative data were presented as percentages. The distribution of data on categorical variables like the patient's clinical parameters was expressed as frequency and percentages. The student's t-test was used to assess the difference in duration of symptoms. Fisher's exact test was used to compare viral clearance between the treatment group and the control group. A two-tailed p-value of less than 0.05 was considered statistically significant.

\section{Results}

We received 120 patients during the study period of which 62 had RT-PCR done on day 14 and 15 following national guidelines. Amongst those 62 patients, 29 of them were symptomatic without any comorbidities and were enrolled in our study as per protocol. Due to the unpredictable efficacy of chloroquine in COVID-19, it was decided that chloroquine would only be administered to patients without comorbidities. In accordance with institutional guidelines, we treated 12 of them with CQ in addition to standard care. In the other group (17 out of 29), patients were treated with standard care as treatment protocol changed (Figure 1).

Baseline demographic data of both groups is presented in Table 1. There were 29 patients in the study population with a mean age of 45.54 years (SD 15.3 years). There was a predominance of males in the study population. $24.13 \%$ of the study participants were smokers. Major symptoms included cough (71.4\%), fever (59.52\%), headache (14.2\%), and hemoptysis (9.5\%).

Among the disease-related complications, 2 patients (6.8\%) had developed hypoxemic respiratory failure. No patient had developed adult respiratory distress syndrome, septic shock, renal failure, liver failure, or multi-organ dysfunction syndrome. 2 out of 12 (16.6\%) patients in the CQ-treated group developed diarrhea.

The duration of symptoms among the CQ-treated group (6.3 \pm 2.7 days) was signifi- 


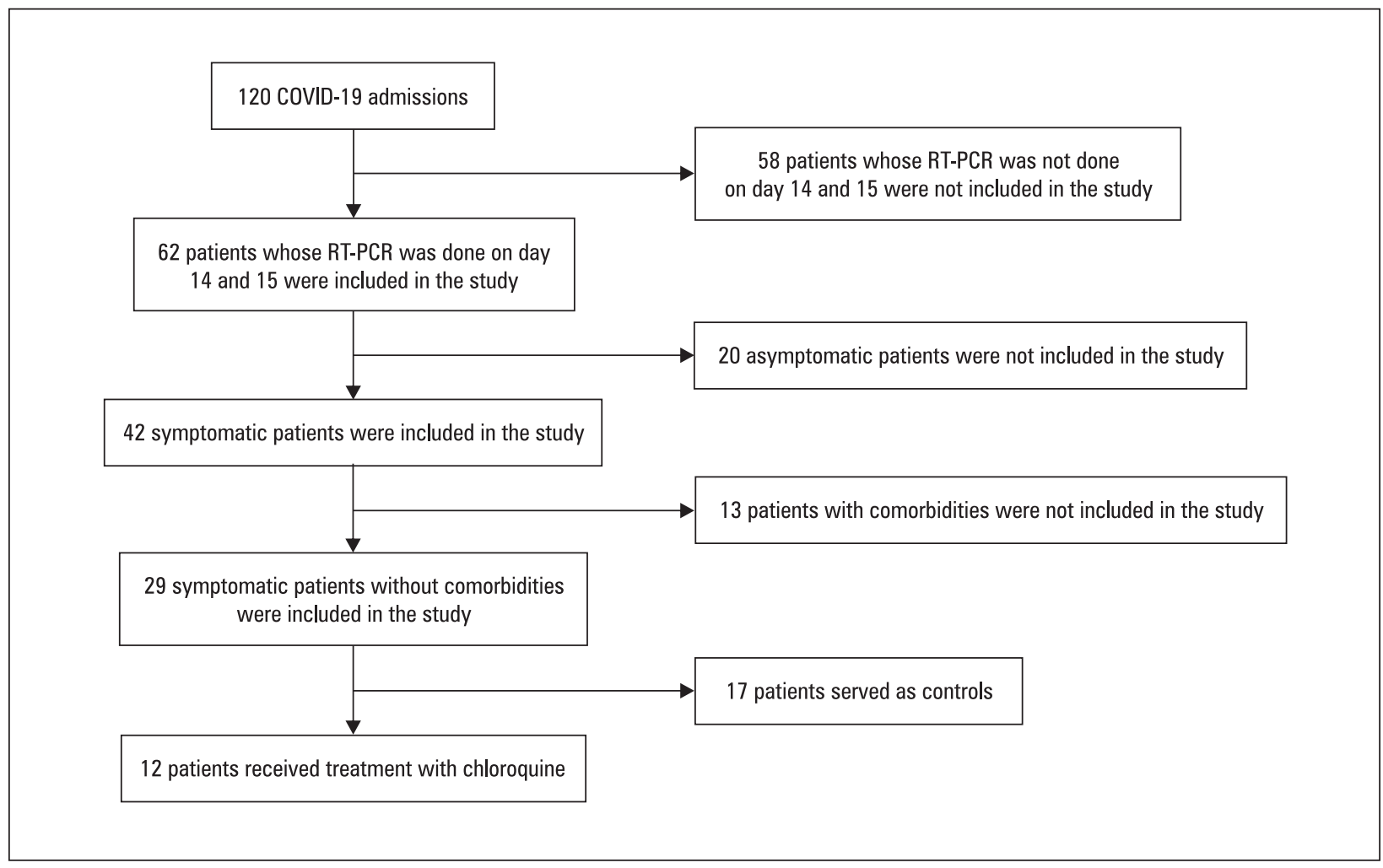

Figure 1. Allocation of participants in the study

Table 1. Baseline demographic and clinical characteristics of patients in the study group and in the control group

\begin{tabular}{|c|c|c|c|}
\hline Characteristics & $\begin{array}{l}\text { Treated group } \\
\text { (n= }=12) \\
\text { Number }(\%)\end{array}$ & $\begin{array}{c}\text { Control group } \\
(\mathbf{n}=17) \\
\text { Number }(\%)\end{array}$ & $\mathrm{p}$ value \\
\hline Males & $9(75 \%)$ & $14(82.3 \%)$ & 0.669 \\
\hline Females & $3(25 \%)$ & $3(17.7 \%)$ & 0.669 \\
\hline Smokers & $2(16.7 \%)$ & $5(29.4 \%)$ & 0.664 \\
\hline Mean age, years [SD] & $41.25(18.04)$ & $47.64(15.30)$ & 0.6 \\
\hline Mean duration of symptoms in days [SD] & $6.3(2.7)$ & $8.9(2.2)$ & 0.009 \\
\hline RT-PCR conversion rate on day 14 and 15 & $10(83.3)$ & $16(94.4)$ & 0.521 \\
\hline
\end{tabular}

cantly (p-value $=0.009$ ) lower than that of the control group (8.9 \pm 2.2 days) (Figure 2).

In our study, RT-PCR conversion on day 15 was similar in both groups ( $\mathrm{p}$-value $>0.05$ ) with conversion seen in 10 out of 12 patients on CQ (83.3\%) compared to 16 out of 17 in the control group (94.4\%) (Figure 3).

\section{Discussion}

In the current pandemic situation, there is a relative scarcity of high-quality reliable data on what constitutes optimal patient care in COVID-19. Drug therapy in COVID-19 remains important as the global treatment recommendations were largely based on empiric evidence and unpowered studies. Specific therapies which are currently considered for treatment include remdesivir, favipiravir, CQ/hydroxychloroquine, interleukin-6 pathway inhibitors, convalescent plasma, favipravir, interferon-beta etc. The United States Food and Drug Administration has authorized conditional use of remdesivir for hospitalized children and adults with severe COVID-19. However, its anti-viral effect has only been proven in vitro and its use is supported by only a few randomized clinical trials [12].

CQ and hydroxychloroquine are currently being considered for the treatment and post-ex- 


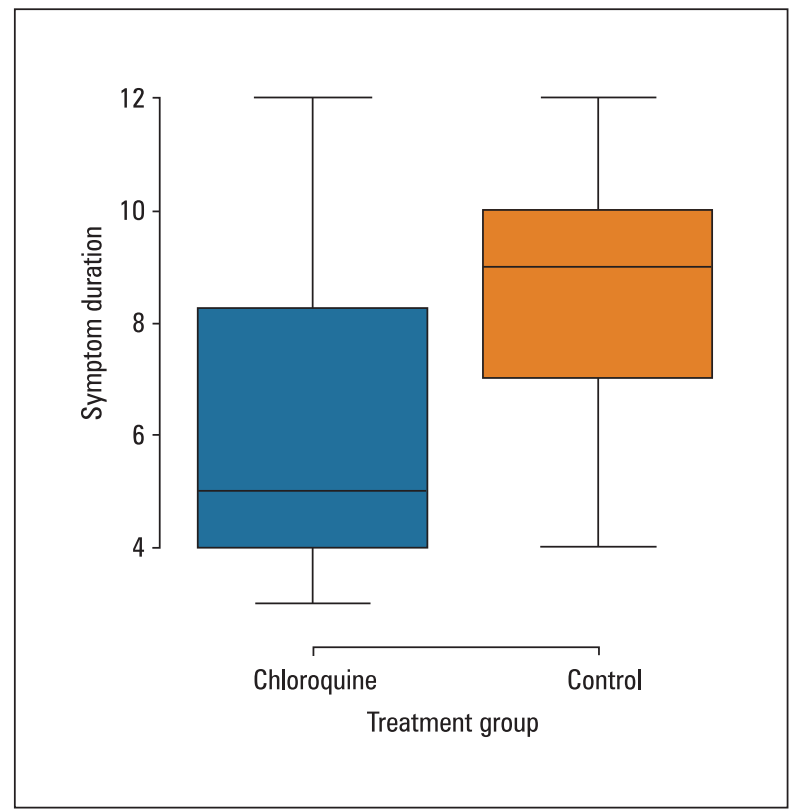

Figure 2. Box-plot showing the distribution of time-to-symptom resolution between the $\mathrm{C} Q$ and control arms

posure prophylaxis of COVID-19 due to its theoretical antiviral and immunomodulatory activity. CQ increases intracellular $\mathrm{pH}$, reduces $\mathrm{T}$ cell activation, impedes the pro-inflammatory signaling pathway, and attenuates the production of TNF-alpha and interleukin-1. CQ was also found to inhibit the key steps of coronavirus entry into cells via angiotensin-converting enzyme 2 (ACE) receptor binding and membrane fusion $[13,14]$. CQ has been used extensively in malaria treatment especially in a malaria-endemic country like India, and it has been proven to have an excellent safety profile. This study is the first reported experience of CQ use for COVID-19 in India.

In our study, RT-PCR conversion on day 14 and 15 was similar in both groups (p-value $>0.05$ ). Although Guatret et al showed early viral conversion in their study involving the use of CQ/HCQ, our results differed from this as we could not do serial RT-PCR testing owing to resource limitation inherent to a developing country [14]. Our result is concordant with the findings of Tang et al. who assessed the efficacy and safety of HCQ in 150 Chinese patients where they found that the negative conversion of RT-PCR was similar for their standard care group as well as the hydroxychloroquine group [15].

The duration of symptoms among the treated group was significantly lower than that of the control group ( $p$-value $=0.009)$. There were no known studies in the literature that had reported time-to-symptom resolution. Our results suggest

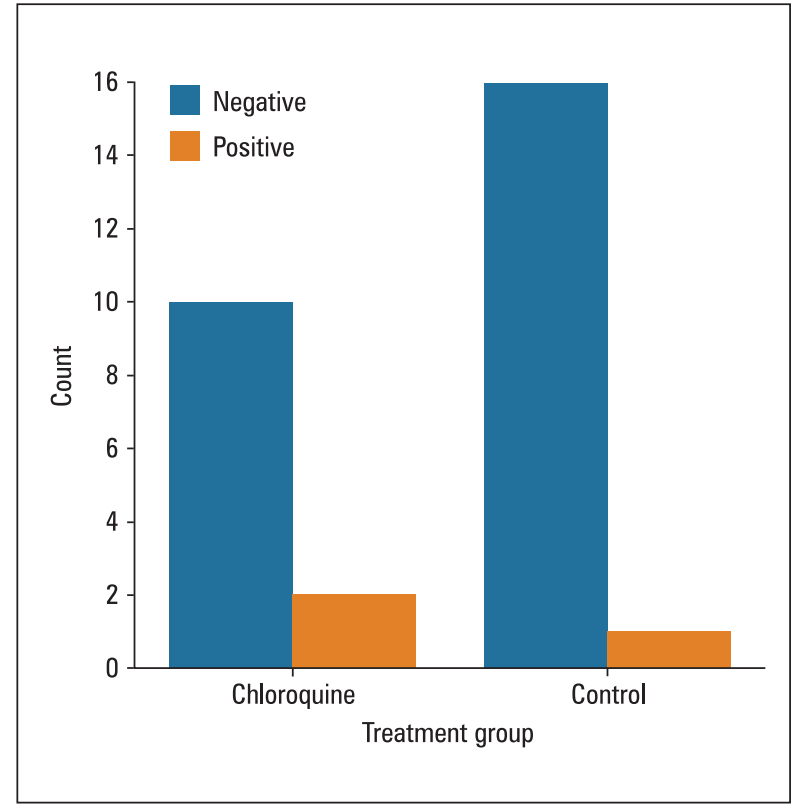

Figure 3. Bar plot showing RT-PCR conversion rate at the end of 2 weeks of hospitalization

that, although CQ is unable to cause an early negative PCR conversion in patients, it assists in early symptom relief.

In a study published by van den Broek et al regarding the safety profile and adverse reactions, QTc interval prolongation greater than 500 milliseconds was seen in 22 of 95 patients (25\%) [16]. In our study, the only adverse event related to drug therapy was diarrhea in 2 out of 12 patients. Tang et al also reported that diarrhea was the most common adverse event noted [15]. Due to known drug interactions between hydroxychloroquine and azithromycin, digoxin, and metoprolol, we did not assess for these confounding factors in our study which may be elicited in larger trials.

Our results from the use of CQ in COVID-19 suggest a limited albeit safe role in early symptom resolution in COVID-19. A shortened duration of symptoms with CQ might decrease the infectivity of the disease as aerosols generated by coughing are an important source of spread in COVID-19.

\section{Strengths and limitations}

This study, being the first reported experience of the use of CQ in the management of COVID-19 in India, and being bound by institutional protocol in COVID-19 management, could only recruit a limited number of patients. However, since we could not find any larger studies from India regarding the use of $\mathrm{HCQ} / \mathrm{CQ}$, we 
proceeded with reporting our limited experience with it. Other limitations included the fact that this was a single center design with limited long term follow up. A shortened duration of symptoms with CQ might decrease the infectivity of the disease as aerosols generated by coughing are an important source of spread in COVID-19.

\section{Conclusion}

The duration of symptoms among the treated group (with CQ) was significantly lower than that of the control group, though RT-PCR conversion was not significantly different between the two. CQ was used without serious adverse events. Larger and multicenter trials are needed to establish its definitive role in COVID-19.

\section{Conflict of interest}

None declared.

\section{References:}

1. Lu R, Zhao X, Li J, et al. Genomic characterisation and epidemiology of 2019 novel coronavirus: implications for virus origins and receptor binding. Lancet. 2020; 395(10224): 565-574, doi: 10.1016/S0140-6736(20)30251-8, indexed in Pubmed: 32007145.

2. WHO. Coronavirus disease (COVID-2019) situation reports. 2020. https://www.who.int/emergencies/diseases/novel-coronavirus-2019/situation-reports. [Last accessed 28.03.2020].

3. Wahba L, Jain N, Fire AZ, et al. A new coronavirus associated with human respiratory disease in China. Nature. 2020; 579(7798): 265-269, doi: 10.1038/s41586-020-2008-3, indexed in Pubmed: 32015508.

4. Zhu Na, Zhang D, Wang W, et al. A novel coronavirus from patients with pneumonia in China, 2019. N Engl J Med. 2020; 382(8): 727-733, doi: 10.1056/NEJMoa2001017, indexed in Pubmed: 31978945.

5. Liu Z, Xiao X, Wei X, et al. Composition and divergence of coronavirus spike proteins and host ACE2 receptors predict potential intermediate hosts of SARS-CoV-2. J Med Virol. 2020; 92(6): 595-601, doi: 10.1002/imv.25726, indexed in Pubmed: 32100877 .

6. Guan WJ, Ni ZY, Hu Yu, et al. Clinical characteristics of coronavirus disease 2019 in china. N Engl J Med. 2020; 382(18):
1708-1720, doi: 10.1056/NEJMoa2002032, indexed in Pubmed: 32109013.

7. Zumla A, Chan JFW, Azhar EI, et al. Coronaviruses - drug discovery and therapeutic options. Nat Rev Drug Discov. 2016; 15(5): 327-347, doi: 10.1038/nrd.2015.37, indexed in Pubmed: 26868298.

8. Zhonghua Jie He He Hu Xi Za Zhi. Multicenter Collaboration Group of Department of Science and Technology of Guangdong Province and Health Commission of Guangdong Province for chloroquine in the treatment of novel coronavirus pneumoniaExpert consensus on chloroquine phosphate for the treatment of novel coronavirus pneumonia [in Chinese], 43 (2020).

9. Gao J, Tian Z, Yang Xu. Breakthrough: Chloroquine phosphate has shown apparent efficacy in treatment of COVID-19 associated pneumonia in clinical studies. Biosci Trends. 2020; 14(1): 72-73, doi: $10.5582 /$ bst.2020.01047, indexed in Pubmed: 32074550 .

10. Savarino A, Trani LDi, Donatelli I, et al. New insights into the antiviral effects of chloroquine. The Lancet Infectious Diseases. 2006; 6(2): 67-69, doi: 10.1016/s1473-3099(06)70361-9.

11. Chinese Clinical Trial Registry. Available at: www.chictr.org. cn/ searchproj.aspx?title=\%E6\%B0\%AF\%E5\%96\%B9\&offi cialname $=\&$ subjectid $=\&$ secondaryid $=\&$ applier $=\&$ study leader $=$ dicalcommitteesanction $=\&$ sponsor $=\&$ studya ilment $=\&$ studyailmentcode $=\&$ studytype $=0 \&$ studystage $=$ $0 \&$ studydesign $=0 \&$ minstudyexecutetime $=\&$ maxstudyexe cutetime $=\&$ recruitmentstatus $=0 \&$ gender $=0 \&$ agreetosign $=\&$ secsponsor $=\circledR$ no $=\circledR$ status $=0 \&$ country $=\&$ prov in $\mathrm{ce}=\&$ city $=$ \&institution $=$ \&institutionlevel $=\&$ measure $=$ \&intercode $=\&$ sourceofspend $=\&$ createyear $=0$ \&isupload $\mathrm{rf}=$ \&whetherpublic $=\&$ btngo $=$ btn\&verifycode $=\&$ page $=1$. Last accessed: 09.03.2020]. .

12. Agostini ML, Andres EL, Sims AC, et al. Coronavirus susceptibility to the antiviral remdesivir (GS-5734) is mediated by the viral polymerase and the proofreading exoribonuclease. mBio. 2018; 9(2), doi: 10.1128/mBio.00221-18, indexed in Pubmed: 29511076.

13. Liu Y, Zhang C, Huang F, et al. 2019-novel coronavirus (2019$\mathrm{nCoV}$ ) infections trigger an exaggerated cytokine response aggravating lung injury. Available at: http://chinaxiv.org/ abs/202002.00018. [Last accessed: 01.04.2020].

14. Rosendaal FR, Gautret P, Lagier JC, et al. Hydroxychloroquine and azithromycin as a treatment of COVID-19: results of an open-label non-randomized clinical trial. Int J Antimicrob Agents. 2020; 56(1): 105949, doi: 10.1016/j.ijantimicag.2020.105949, indexed in Pubmed: 32205204.

15. Tang W, Cao Z, Han M, et al. Hydroxychloroquine in patients with mainly mild to moderate coronavirus disease 2019: open label, randomised controlled trial. BMJ. 2020; 369: m1849, doi: 10.1136/bmj.m1849, indexed in Pubmed: 32409561.

16. van den Broek MPH, Möhlmann JE, Abeln BGS, et al. Chloroquine-induced QTc prolongation in COVID-19 patients. Neth Heart J. 2020; 28(7-8): 406-409, doi: 10.1007/s12471-02001429-7, indexed in Pubmed: 32350818. 